\title{
Lipossarcoma do Cordão Espermático
}

\section{Spermatic Cord Liposarcoma}

\author{
Raquel Catarino ${ }^{1 *}$, André Cardoso', Carlos Ferreira1, Diogo Pereira1, Tiago Correia', Manuel Cerqueira1, Frederico Carmo \\ Reis ${ }^{1}$, Paulo Costa ${ }^{2}$, Rocha Almeida ${ }^{3}$, Rui Prisco ${ }^{1}$
}

\section{Resumo}

Introdução: O lipossarcoma do cordão espermático é uma doença rara, com poucos casos descritos. Apresentamos dois casos clínicos de doentes com lipossarcoma do cordão espermático.

Caso Clínico: Caso 1: Doente de 76 anos, avaliado por uma massa inguinal esquerda indolor. O estudo imagiológico evidenciou uma massa sólida, com cerca de $38 \times 31 \mathrm{~cm}$ na região inguinal esquerda. Caso 2: Doente de 55 anos, avaliado por uma massa escrotal esquerda indolor. Imagiologicamente apresentava lesão sólida com $28 \times 27 \mathrm{~cm}$, sugestiva de neoformação na dependência do epidídimo esquerdo.

Ambos os doentes foram submetidos a orquidectomia radical com excisão local alargada. O exame histopatológico revelou lipossarcoma nas duas situações, tendo sido efetuado tratamento complementar com radioterapia adjuvante.

No seguimento efetuado, oito anos no caso 1 e quatro anos no caso 2, não ocorreu recidiva neoformativa locorregional ou à distância.

Conclusão: O tratamento recomendado do lipossarcoma do cordão espermático é a orquidectomia radical com excisão local alargada. A radioterapia adjuvante reduz o número de recorrências locorregionais e torna-se necessário um período longo de seguimento destes doentes, dada a elevada probabilidade de recorrência.

Palavras-chave: Cordão Espermático; Lipossarcoma; Neoplasias dos Genitais Masculinos; Orquidectomia.

\section{Introdução}

Os tumores paratesticulares malignos são raros, com uma frequência estimada de $7 \%-10 \%$ de todos os tumores intraescrotais. Na maioria dos casos, em cerca de $75 \%$, estão localizados no cordão espermático. As lesões malignas do cordão são maioritariamente sarcomas, dos quais cerca de $5 \%$ a $7 \%$ são lipossarcomas. ${ }^{1,2}$

O lipossarcoma paratesticular, descrito pela primeira vez em 1952, é uma neoplasia maligna de tecido adiposo originada a partir de células mesenquimatosas primitivas do cordão

${ }^{1}$ Serviço de Urologia, Hospital Pedro Hispano, Matosinhos, Portugal ${ }^{2}$ Serviço de Radioterapia, Instituto CUF-Porto, Porto, Portugal ${ }^{3}$ Serviço de Radiologia, Hospital CUF-Porto, Porto, Portugal

\section{Abstract}

Introduction: Spermatic cord liposarcomas are rare diseases, with only a few reported studies published. In this study we present 2 clinical cases of spermatic cord liposarcoma. Case Report: Case 1: A 76-years-old man was evaluated for a painless left inguinal mass. Imaging studies demonstrated a solid mass, of $38 \times 31 \mathrm{~cm}$, located in the left inguinal region.

Case 2: A 55-years-old men, evaluated for a painless left scrotal mass, with imaging studies demonstrating a solid lesion of $28 \times 27 \mathrm{~mm}$ near the left testicle.

Both patients underwent left radical orchiectomy, with wide local excision. Histological examination revealed liposarcoma in both situations and the patients performed adjuvant radiotherapy.

The patients remain free of disease during follow-up, of eight years in case 1 and four years in the case 2 .

Conclusion: The recommended treatment of spermatic cord liposarcomas is radical orchiectomy with wide local excision. Adjuvant radiotherapy can reduce de incidence of local recurrence. Considering the recurrence probability, a long follow-up period is recommended for these patients.

Keywords: Genital Neoplasms, Male; Liposarcoma; Orchiectomy; Spermatic Cord.

espermático. ${ }^{1}$ Existem apenas cerca de 200 casos de lipossarcoma do cordão espermático publicados. A maioria dos casos é diagnosticada na idade adulta, mais comummente entre os 50 e 60 anos. Estes tumores são frequentemente insidiosos e assintomáticos, apresentam-se habitualmente como uma massa escrotal ou inguinal indolor e de crescimento lento. O diagnóstico diferencial deve incluir hérnia inguinal ou escrotal, hidrocelo, varicocelo, epididimite e tumor testicular. A apresentação pode ser retardada, com diagnóstico mais tardio, dado o seu crescimento lento e ausência de sintomas. ${ }^{1,3}$

Dada a sua raridade, existem poucos estudos publicados sobre a avaliação diagnóstica e orientação terapêutica destes doentes. Descrevemos dois casos clínicos de doentes com lipossarcoma do cordão espermático, considerando a 


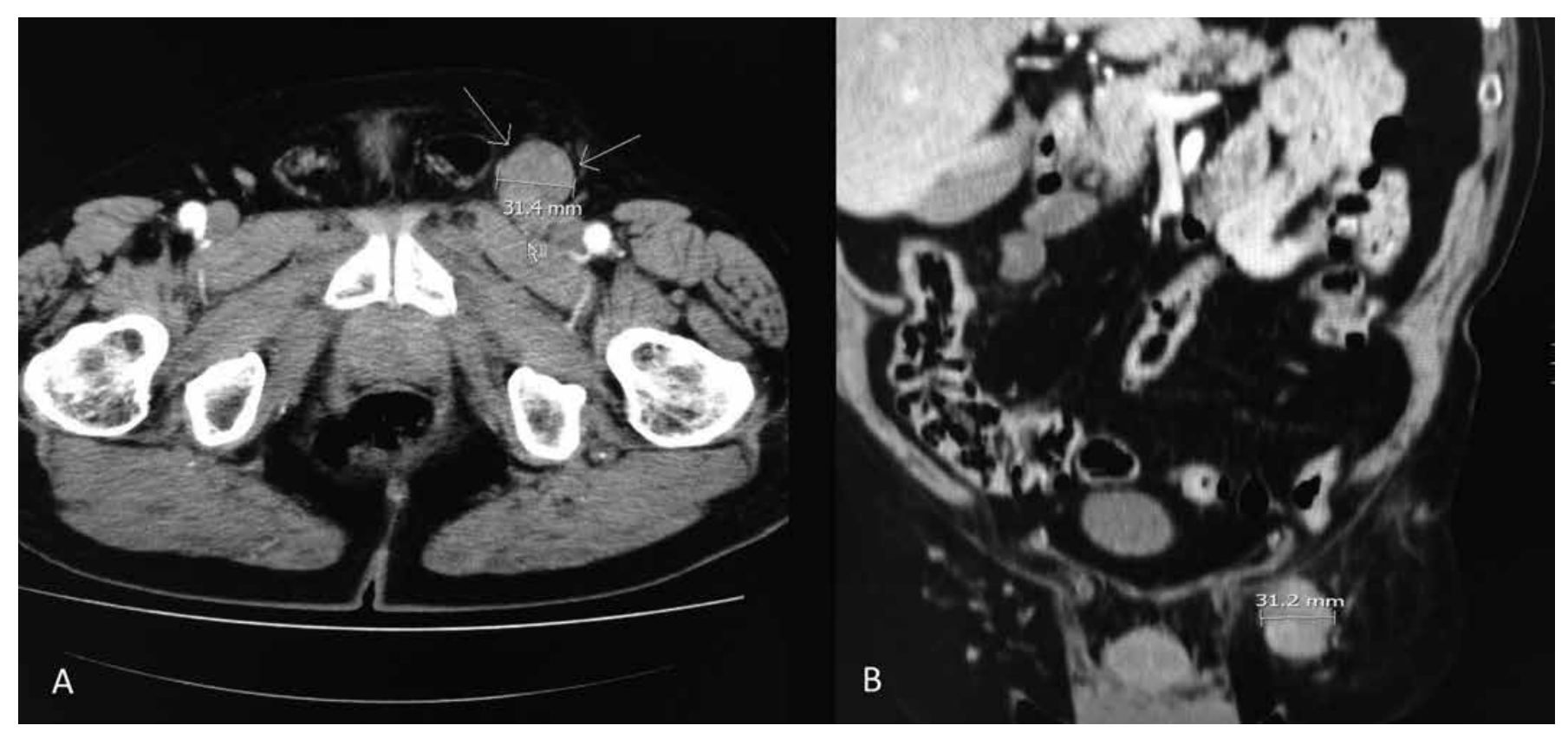

Figura 1: Tomografia computorizada a evidenciar a presença de um lipossarcoma na região inguinal esquerda. a) corte axial; b) corte coronal.

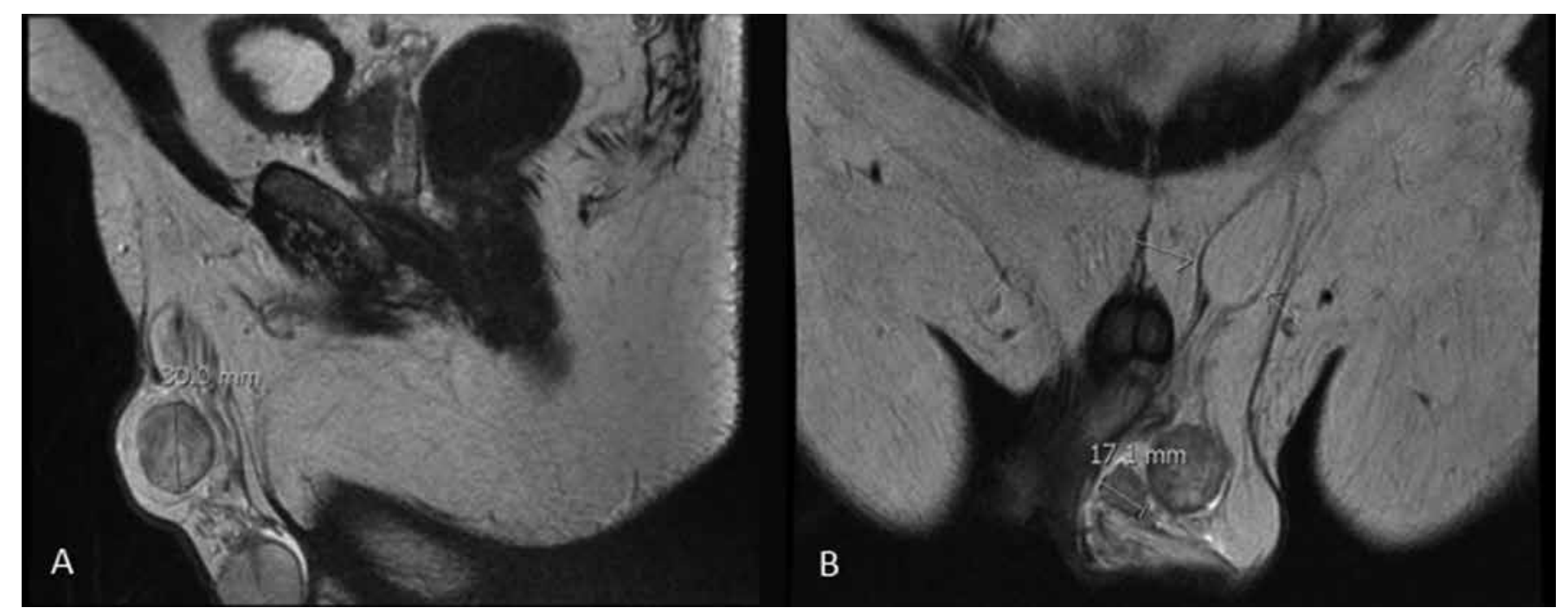

Figura 2: Ressonância magnética da região escrotal a demonstrar a presença de um lipossarcoma à esquerda. a) corte sagital; b) corte coronal.

apresentação clínica, achados imagiológicos e opções de tratamento.

\section{Casos Clínicos}

\section{CASO 1}

Doente de 76 anos, sem antecedentes de relevo, foi avaliado por uma tumefação inguinal esquerda indolor, com crescimento lento e progressivo ao longo de vários meses. Ao exame objetivo apresentava uma massa sólida, móvel e irregular na região inguinal esquerda, sem alterações palpáveis a nível testicular. Realizou estudo analítico com avaliação de marcadores tumorais, nomeadamente alfa-fetoproteína, fração beta da hormona gonadotrófica (B-HcG) e DHL, que não apresentava alterações. A ecografia mostrou três imagens ovaladas, hipoecogénicas, a maior com 33 mm de diâmetro na região inguinal esquerda.

Foi realizada biópsia aspirativa das lesões, tendo sido inconclusiva, evidenciando a presença de agrupamentos de células fusiformes, favorecendo o diagnóstico de neoplasia mesenquimatosa.

A tomografia computorizada (TC) abdomino-pélvica revelou uma massa sólida, com cerca de $38 \times 31 \mathrm{~mm}$ na região inguinal esquerda, sem evidência de metastização. Apresentava também hérnia inguinal bilateral, embora apenas de tecido adiposo, mais proeminente à esquerda (Fig.s 1 a e b). 


\section{CASO 2}

Doente de 55 anos, avaliado por uma tumefação escrotal esquerda, indolor, de crescimento lento. Ao exame objetivo apresentava uma massa sólida, irregular na região escrotal, aparentemente individualizada do testículo esquerdo. A ecografia mostrou uma lesão nodular sólida, irregular, com 28 x $27 \mathrm{~cm}$, hipervascularizada, sugestiva de neoformação na dependência do epidídimo esquerdo.

Realizou também estudo analítico com avaliação de marcadores tumorais, nomeadamente alfa-fetoproteína, B-HcG e DHL, que não apresentavam alterações. Efetuou ressonância magnética (RM) abdomino-pélvica que revelou uma formação nodular com $3 \mathrm{~cm}$ de maior eixo, cranialmente ao testículo e epidídimo esquerdos, vascularizada, bem delimitada, sem invasão de estruturas adjacentes e sem evidência de metastização (Fig.s 2 a e b).

Ambos os doentes foram submetidos a orquidectomia radical e excisão local alargada, com ressecção em bloco da massa e estruturas adjacentes do cordão espermático. O diagnóstico anatomopatológico foi de lipossarcoma nas duas situações, do tipo histológico misto (mixóide e de células redondas) no caso 1 e indiferenciado no caso 2, com margens cirúrgicas negativas em ambos os casos.

Em consulta de grupo multidisciplinar foi decidida a realização de tratamento complementar com radioterapia adjuvante nos dois doentes, tendo realizado a dose recomendada de 60 Gy durante 6 semanas.

No seguimento efetuado, oito anos no caso 1 e quatro anos no caso 2, os doentes não apresentaram sinais de recidiva local ou à distância.

Ambos os doentes concordaram em participar neste estudo, com consentimento informado livre esclarecido, de acordo com a declaração de Helsínquia.

\section{Discussão}

Os tumores paratesticulares são neoplasias raras, sendo na sua maioria tumores benignos (70\% - $80 \%)$, mais comummente lipomas. ${ }^{1,2,4}$

Os lipossarcomas do cordão espermático são responsáveis por apenas $0,03 \%$ de todos os tumores urológicos, de acordo com a escassez de estudos publicados sobre esta temática, que compreendem principalmente estudos de casos clínicos ou pequenas séries de casos. . $^{5-8}$

A maioria dos lipossarcomas do cordão espermático parece surgir de novo, sendo que os lipomas simples, que constituem cerca de $80 \%$ de todas as neoformações, transformam-se muito raramente em sarcomas. A maioria dos casos é originada diretamente no cordão espermático, mas podem ter origem no retroperitoneu e desenvolver-se na região inguinal, envolvendo o cordão espermático..$^{5,9}$

Estes tumores apresentam-se habitualmente como uma massa inguinal ou inguino-escrotal de crescimento indolor, podendo ser facilmente diagnosticados como hérnias inguinais, lipomas, hidrocelos ou epididimites crónicas. ${ }^{3}$

Relativamente aos exames auxiliares de diagnóstico, a ecografia pode ser útil na caracterização da lesão e avaliação do cordão espermático, testículo e anexos, evidenciando geralmente uma massa sólida, hiperecogénica e heterogénea, separada do testículo. No caso de suspeita de neoplasia, as lesões podem ser avaliadas com tomografia computorizada ou ressonância magnética para estudo mais detalhado e estadiamento. ${ }^{10}$

Os tipos histológicos mais comuns dos tumores paratesticulares malignos incluem o lipossarcoma (20\% - 56\%), leiomiossarcoma (19\% - 32\%), histiocitoma fibroso maligno (13\%) e rabdomiossarcoma (11\% - 24\%). De acordo com a histologia, os lipossarcomas podem ser divididos em subtipos: bem-diferenciado, mixóide, de células redondas, pleomórfico, indiferenciado ou misto. Os subtipos de baixo grau incluem os tumores bem diferenciados, que apresentam baixo potencial de metastização, podendo, no entanto, ser localmente invasivos. Os subtipos de alto grau (pleomórficos, de células redondas e indiferenciados, como são os casos apresentados), são mais raros e estão associados a uma maior taxa de recorrência e metastização hematogénea. ${ }^{11}$

Dada a sua raridade, as opções de tratamento mais indicadas para os lipossarcomas permanecem ainda por esclarecer. A abordagem terapêutica preconizada inclui orquidectomia radical com excisão local alargada, tendo como objetivo a ressecção completa da lesão. No entanto, devido a dificuldades anatómicas, estes tumores apresentam-se difíceis de excisão alargada, com margens cirúrgicas frequentemente positivas. Por outro lado, os sarcomas tendem a infiltrar tecidos locais, aumentado a dificuldade de uma ressecção adequada. Assim, a recorrência locorregional permanece um problema comum. Coleman e colaboradores reportaram que aproximadamente um terço dos doentes que foram submetidos a re-excisão, sem doença clinicamente evidente, apresentavam tecido tumoral residual. ${ }^{5}$

A extensão de excisão de tecido apropriado permanece ainda controversa, e a excisão simples parece ser inadequada, com elevadas taxas de doença residual. Assim, estratégias cirúrgicas mais agressivas são recomendadas no tratamento destes doentes, envolvendo a excisão em bloco de todos os tecidos envolventes potencialmente envolvidos e re-excisão em caso de recorrência da doença. ${ }^{5}$

Geralmente não existe indicação para linfadenectomia locorregional, uma vez que a disseminação ganglionar é rara. ${ }^{5,12}$

No que concerne ao prognóstico, os fatores mais importantes incluem a ressecção completa da massa tumoral em bloco, o tipo e o grau histológicos e o tamanho do tumor.

Têm sido descritas taxas de recorrência locorregional em cerca de $50 \%$ dos casos aos 10 anos. Dada a probabilidade 
de recorrência local, tem sido proposta a realização de radioterapia adjuvante no sentido de diminuir a incidência de recidiva locorregional. Por outro lado, a radioterapia tem também sido utilizada quando há margens positivas. O lipossarcoma de células mixóides parece ser o mais sensivel à radioterapia. O papel da quimioterapia não está ainda estabelecido no tratamento dos sarcomas do cordão espermático, dada a quimiorresistência dos tipos histológicos mais comuns. ${ }^{12-15}$

Considerando a elevada taxa de recorrência, nomeadamente tardia, preconiza-se um seguimento prolongado destes doentes, estando recomendado o follow-up por pelo menos 10 anos. A sobrevivência destes doentes aos 5 anos parece ser de aproximadamente $75 \%{ }^{5}$

Caso não seja considerada a hipótese de sarcoma no diagnóstico inicial, pré-operatório, a cirurgia radical não é realizada, o que aumenta a morbilidade do doente pela necessidade de intervenções cirúrgicas posteriores, aumenta a probabilidade de requerer tratamento adjuvante, com radioterapia, e há uma associação com pior prognóstico.

\section{Responsabilidades Éticas}

Conflitos de Interesse: Os autores declaram a inexistência de conflitos de interesse na realização do presente trabalho.

Fontes de Financiamento: Não existiram fontes externas de financiamento para a realização deste artigo.

Protecção de Pessoas e Animais: Os autores declaram que os procedimentos seguidos estavam de acordo com a Declaração de Helsínquia da Associação Médica Mundial. Confidencialidade dos Dados: Os autores declaram ter seguido os protocolos do seu centro de trabalho acerca da publicação dos dados de doentes.

\section{Ethical Disclosures}

Conflicts of interest: The authors report no conflict of interest. Funding sources: No subsidies or grants contributed to this work.

Financing Support: This work has not received any contribution, grant or scholarship. Protection of Human and Animal Subjects: The authors declare that the procedures followed were in accordance with the regulations of the relevant clinical research ethics committee and with those of the Code of Ethics of the World Medical Association (Declaration of Helsinki).

Confidentiality of Data: The authors declare that they have followed the protocols of their work center on the publication of patient data.

\section{${ }^{*}$ Corresponding Author $/{ }^{*}$ Autor Correspondente:}

Raquel Catarino

Serviço de Urologia

Hospital Pedro Hispano

R. Dr. Eduardo Torres

4464-513 Senhora da Hora Portugal

E-mail: raquelcatarino@gmail.com

Recebido/Received: 2017-11-30

Aceite/Accepted: 2018- 04-17

\section{REFERÊNCIAS}

1. Fitzgerald S, Maclennan GT. Paratesticular liposarcoma. J Urol. 2009; 181: $331-2$.

2. Priemer DS, Trevino K, Chen S, Ulbright TM, Idrees MT. Paratesticular soft-tissue masses in orchiectomy specimens: a 17-year survey of primary and incidental cases from one institution. Int J Surg Pathol. 2017; 25: 480-7.

3. Chintamani, Tandon M, Khandelwal R, Jain S, Narayan N, Kumar Y, et al. Liposarcoma of the spermatic cord: a diagnostic dilemma. JRSM Short Rep. 2010; $1: 49$

4. Mondaini N, Palli D, Saieva C, Nesi G, Franchi A, Ponchietti R, et al. Clinical characteristics and overall survival in genitourinary sarcomas treated with curative intent: a multicenter study. Eur Urol. 2005; 47: 468-73.

5. Coleman J, Brennan MF, Alektiar K, Russo P. Adult spermatic cord sarcomas: management and results. Ann Surg Oncol. 2003; 10: 669-75.

6. Li F, Tian R, Yin C, Dai X, Wang H, Xu N, et al. Liposarcoma of the spermatic cord mimicking a left inguinal hernia: a case report and literature review. World J Surg Oncol. 2013; 11: 18

7. Chalouhy C, Ruck JM, Moukarzel M, Jourdi R, Dagher N, Philosophe B. Current management of liposarcoma of the spermatic cord: A case report and review of the literature. Mol Clin Oncol. 2017; 6: 438-40.

8. Panus A, Mesina C, Plesea IE, Dr goescu PO, Turcitu N, Maria C, et al. Paratesticular liposarcoma of the spermatic cord: a case report and review of the literature. Rom J Morphol Embryol. 2015; 56: 1153-7.

9. Valeshabad AK, Walsh A, Lloyd GL. An important mimic of inguinal hernia. Urology. 2016; 97: e11.

10. Cardenosa G, Papanicolaou N, Fung CY, Tung GA, Yoder IC, Althausen AF et al. Spermatic cord sarcomas: sonographic and CT features. Urol Radiol. 1990; 12: 163-7.

11. Khoubehi B, Mishra V, Ali M, Motiwala H, Karim O. Adult paratesticular tumours. BJU Int. 2002; 90: 707-15

12. Bonvalot S, Miceli R, Berselli M, Causeret S, Colombo C, Mariani L, et al. Aggressive surgery in retroperitoneal soft tissue sarcoma carried out at high-volume centers is safe and is associated with improved local control. Ann Surg Oncol. 2010; 17: 1507-14.

13. Bonvalot S, Rivoire M, Castaing M, Stoeckle E, Le Cesne A, Blay JY, et al. Primary retroperitoneal sarcomas: a multivariate analysis of surgical factors associated with local control. J Clin Oncol. 2009; 27: 31-7.

14. Ballo MT, Zagars GK, Pisters PW, Feig BW, Patel SR, von Eschenbach AC Spermatic cord sarcoma: outcome, patterns of failure and management. $J$ Urol. 2001; 166: 1306-10

15. Crago AM and Dickson MA. Liposarcoma: multimodality management and future targeted therapies. Surg Oncol Clin N Am. 2016; 25: 761-73. 